\title{
Effect of normal stress under an excitation in poroelastic flat slabs
}

\author{
B. Sandhya $\operatorname{Rani}^{1}$, T. Ramesh $^{2}$, P. Malla Reddy ${ }^{{ }^{*}}$ \\ ${ }^{1 *}$ Department of Mathematics, Kakatiya University, Warangal , INDIA \\ ${ }^{2}$ Department of Mathematics, Kakatiya Degree College, Warangal , INDIA \\ *Corresponding Author: e-mail: mperati@yahoo.com, Tel +91-9866578585
}

\begin{abstract}
Biot's poroelastic theory is employed to investigate stresses under an excitation in an infinite poroelastic slab of arbitrary thickness. Both for pervious and impervious surfaces, the radial normal stress is obtained, and in the neighbourhood of the centre, the same is computed as function of thickness of the slab. Numerical data is presented graphically and then discussed. It is seen that nature of surface, mass coupling parameter and elastic constants (Lame constants) have greater influence over radial normal stress.
\end{abstract}

Keywords: Flat slab, radial normal stress, pervious surface, impervious surface.

\section{Introduction}

Flat slabs are most commonly used in structures such as railway stations, bus stations, exhibition halls, and large structures, like, high towers, telecom masts, etc. Such structures when exposed to natural turbulent wind are susceptible to wind induced excitation phenomena. In an earlier study (Davids \& Kumar, 1957), wave propagation in elastic flat slabs under excitation is carried out and even compared with experimental data. However, above structures are poroelastic in nature, hence they are to be investigated using the theory of poroelasticity. Waves of axial symmetry in poroelastic cylindrical structures are studied in cylindrical coordinate system wherein boundaries go with radial coordinate (Reddy \& Tajuddin, 2000; Tajuddin \& Shah, 2007; Tajuddin \&. Shah, 2006; Tajuddin \& Reddy, 2005) in the framework (Biot, 1956). Wave propagation in poroelastic flat slabs wherein boundaries go with the azimutal coordinate is studied in the paper (Reddy \& Tajuddin, 2006). In the said analysis, the frequency equation is investigated for a pervious boundary and an impervious boundary and realized the fact that the nature of boundary and mass coupling parameter influence wave propagation. Earlier studies have not considered excitation phenomena, which is warranted, because of above mentioned applications.

In this paper, the waves under line source sinusoidal excitation are obtained thereby the radial normal stress along thickness of the slab is computed, and seen how the nature of surface and material parameters influence the values. Although, our analysis would confine to a periodic disturbance, because of the Fourier integral theorem, it is possible to extend conclusions to pulse propagation as well.

The rest of the paper is organized as follows. First, the problem is formulated and the boundary conditions are prescribed in section 2. Then in section 3, waves under line source excitation is investigated. The non-dimensionalisation as well as numerical results is discussed in section 4 . Finally, concluding remarks are given.

\section{The Boundary Value Problem}

Consider an infinite poroelastic slab of thickness ' $2 a$ ' excited along a line coinciding with the z-axis in cylindrical polar coordinate system $(r, \theta, z)$. Let the slab be homogeneous and isotropic. The equations of motion of a poroelastic solid (Biot 1956) in presence of dissipation $(b)$ which in terms of displacement vectors are: 


$$
\begin{aligned}
& N \nabla^{2} \vec{u}+\nabla[(A+N) e+Q \in]=\frac{\partial^{2}}{\partial t^{2}}\left(\rho_{11} \vec{u}+\rho_{12} \vec{U}\right)+b \frac{\partial}{\partial t}(\vec{u}-\vec{U}), \\
& \nabla[Q e+R \in]=\frac{\partial^{2}}{\partial t^{2}}\left(\rho_{12} \vec{u}+\rho_{22} \vec{U}\right)-b \frac{\partial}{\partial t}(\vec{u}-\vec{U}) .
\end{aligned}
$$

In (1), $P(=A+2 N), N, Q, R$ are all poroelastic constants, $\nabla^{2}$ is the Laplacian operator; and $\rho_{i j}$ are mass coefficients, $e \quad$ and $\in$ are solid dilatation and fluid dilatation, respectively. The solid displacement components $(u, 0, w)$ which are functions of $r$, $z$ and $t$ (here the problem is plane strain that is independent of $\theta$ ) which can readily be evaluated from the field equations (Biot 1956) representing steady state harmonic vibrations presented in matrix notation are

$$
\left[\begin{array}{c}
\frac{u}{-J_{1}(k r)} \\
\frac{w}{-J_{0}(k r)}
\end{array}\right]=\left[\begin{array}{ccc}
k \cosh \left(\alpha_{1} z\right) & k \cosh \left(\alpha_{2} z\right) & \beta k \cos (\beta z) \\
\alpha_{1} \sin \left(\alpha_{1} z\right) & \alpha_{2} \sin \left(\alpha_{2} z\right) & -k^{2} \sin (\beta z)
\end{array}\right]\left[\begin{array}{l}
A_{1} \\
B_{1} \\
C_{1}
\end{array}\right] e^{i p t},
$$

where $p$ is the frequency of wave, $k$ is the wavenumber, $A_{1}, B_{1}, C_{1}$ are all constants. $J_{n}(x)$ is a Bessel function of first kind of order $n$ and

$$
\beta=\left(p^{2} V_{3}^{-2}-k^{2}\right)^{1 / 2}, \alpha_{i}=\left(p^{2} V_{i}^{-2}-k^{2}\right)^{1 / 2}, i=1,2 .
$$

In equation (3), $\quad V_{i}(i=1,2,3)$ are dilatational wave velocities of first and second kind, and shear wave velocity, respectively. Using these displacements into stress-displacement relations (Biot 1956) the relevant stresses $\left(\sigma_{i j}\right)$ and liquid pressure $(s)$ are obtained as follows:

$$
\left[\begin{array}{c}
\sigma_{z z} \\
\sigma_{z r} \\
\sigma_{r r} \\
s
\end{array}\right]=\left[\begin{array}{ccc}
-D_{1} \cos \left(\alpha_{1} z\right) J_{0}(k r) & -D_{2} \cos \left(\alpha_{2} z\right) J_{0}(k r) & 2 N \beta k^{2} \cos (\beta z) J_{0}(k r) \\
2 N k \alpha_{1} \sin \left(\alpha_{1} z\right) J_{1}(k r) & 2 N k \alpha_{2} \sin \left(\alpha_{2} z\right) J_{1}(k r) & 2 N\left(\beta^{2}-k^{2}\right) \sin (\beta z) J_{1}(k r) \\
-D_{5} \cos \left(\alpha_{1} z\right) & -D_{6} \cos \left(\alpha_{2} z\right) & -N k^{2} \beta\left(J_{0}(k r)-J_{2}(k r)\right) \beta \cos (\beta z) \\
-D_{3} \cos \left(\alpha_{1} z\right) J_{0}(k r) & -D_{4} \cos \left(\alpha_{2} z\right) J_{0}(k r) & 0
\end{array}\right]\left[\begin{array}{l}
A_{1} \\
B_{1} \\
C_{1}
\end{array}\right] e^{i p t},
$$

where

$$
\begin{aligned}
& D_{1}=2 N \alpha_{1}^{2}+(P-2 N)\left(k^{2}+\alpha_{1}^{2}\right)+Q\left(k^{2}+\alpha_{1}^{2}\right) \delta_{1} \\
& D_{3}=\left(Q+R \delta_{1}\right)\left(k^{2}+\alpha_{1}^{2}\right) \\
& D_{5}=D_{7} J_{0}(k r)+N k^{2} J_{2}(k r) \\
& D_{7}=N k^{2}+(P-2 N)\left(k^{2}+\alpha_{1}^{2}\right)+Q\left(k^{2}+\alpha_{1}^{2}\right) \delta_{1} \\
& \delta_{i}=\sqrt{\frac{m_{11} V_{i}^{2}-P}{m_{22} V_{i}^{2}-R}}, \quad(i=1,2) \\
& m_{11}=\rho_{11}-i b p^{-1}, \quad m_{12}=\rho_{12}+i b p^{-1}, \quad m_{22}=\rho_{22}-i b p^{-1}
\end{aligned}
$$

$D_{2}, D_{4}, D_{6}, D_{8}=$ similar expressions as $D_{1}, D_{3}, D_{5}, D_{7}$ with $\alpha_{1}$ and $\delta_{1}$ replaced by $\alpha_{2}$ and $\delta_{2}$, respectively.

The boundary condition that the front and back surfaces $z=a$ and $z=-a$ for a pervious surface and an impervious surface to be stress free are

$$
\sigma_{z z}+s=0, \sigma_{r z}=0, s=0
$$

(Pervious surface),

and 


$$
\sigma_{z z}+s=0, \sigma_{r z}=0, \frac{\partial s}{\partial z}=0 \quad \text { (Impervious surface). }
$$

Equations (4) and (5) together give a system of three homogeneous equations for the constants $A_{1}, B_{1}, C_{1}$ each for a pervious surface and an impervious surface. In order to obtain a nontrivial solution of this system, the coefficient matrix must be singular. This leads to a frequency equation for both pervious and impervious surfaces. These frequency equations and their limiting cases are investigated in the paper (Malla Reddy \& Tajuddin 2006).

\section{The Excitation}

This section presents the conditions under which a wave represented by radial and azimuthal displacement components can be generated for a pervious surface and an impervious surface. Since the boundaries are stress free, there can be no excitation from the faces of slab. The only remaining source for the wave, which must be radially symmetric, can be a line corresponding to the axis of cylindrical coordinate system. It is interesting to see how these free boundaries are affecting the stress components $\sigma_{r z}$ and $\sigma_{r r}$ pertaining to radial coordinate and presented for both pervious and impervious surfaces given under:

\subsection{Pervious surface}

Because of the boundary conditions, arbitrary constants $A_{1}, B_{1}, C_{1}$ are no longer independent but are connected as follows:

From the third boundary condition, we have $\frac{A_{1}}{B_{1}}=\frac{-D_{4} \cos \left(\alpha_{2} a\right)}{D_{3} \cos \left(\alpha_{1} a\right)}$.

Substituting (6) in the first boundary condition, we obtain

$$
\frac{C_{1}}{B_{1}}=\frac{-D_{4}\left(D_{1}+D_{3}\right) \cos \left(\alpha_{2} a\right)}{2 D_{3} N \beta k^{2} \cos (\beta a)}+\frac{\left(D_{2}+D_{4}\right) \cos \left(\alpha_{2} a\right)}{2 N \beta k^{2} \cos (\beta a)}
$$

Substituting (6) and (7) in the expression for $\sigma_{r r}+S$, we obtain

$$
\begin{aligned}
\sigma_{r r}+s= & B_{1}\left[\frac{D_{4}\left(D_{3}+D_{7}\right) \cos \left(\alpha_{2} a\right) \cos \left(\alpha_{1} z\right)}{D_{3} \cos \left(\alpha_{1} a\right)}-\left(D_{4}+D_{8}\right) \cos \left(\alpha_{2} z\right)+\right. \\
& \left.\left\{\frac{D_{4}\left(D_{1}+D_{3}\right) \cos \left(\alpha_{2} a\right)}{2 D_{3} \cos (\beta a)}-\frac{\left(D_{2}+D_{4}\right) \cos \left(\alpha_{2} a\right)}{2 \cos (\beta a)}\right\} \cos (\beta z)\right] J_{0}(k r) e^{i p t}+ \\
& B_{1} N k^{2}\left[\frac{D_{4} \cos \left(\alpha_{2} a\right) \cos \left(\alpha_{1} z\right)}{D_{3} \cos \left(\alpha_{1} a\right)}-\cos \left(\alpha_{2} z\right)+\left\{\frac{-D_{4}\left(D_{1}+D_{3}\right) \cos \left(\alpha_{2} a\right)}{2 D_{3} N k^{2} \cos (\beta a)}+\right.\right. \\
& \left.\left.\left.\frac{\left(D_{2}+D_{4}\right) \cos \left(\alpha_{2} a\right)}{2 N k^{2} \cos (\beta a)}\right\} \cos (\beta z)\right\}\right] J_{2}(k r) e^{i p t} .
\end{aligned}
$$

Similar expression can be obtained for $\sigma_{r z}$. The case of axial excitation can be dealt with by letting $r \rightarrow 0$ in the expressions for $\sigma_{r r}+s$ and $\sigma_{r z}$. Their values in this case are

$$
\sigma_{r z}=0, \quad \sigma_{r r}+s=B_{1} F_{1} e^{i p t},
$$


where

$$
\begin{gathered}
F_{1}=\left[\frac{D_{4}\left(D_{3}+D_{7}\right) \cos \left(\alpha_{2} a\right) \cos \left(\alpha_{1} z\right)}{D_{3} \cos \left(\alpha_{1} a\right)}-\left(D_{4}+D_{8}\right) \cos \left(\alpha_{2} z\right)+\left\{\frac{D_{4}\left(D_{1}+D_{3}\right) \cos \left(\alpha_{2} a\right)}{2 D_{3} \cos (\beta a)}\right.\right. \\
\left.\left.-\frac{\left(D_{2}+D_{4}\right) \cos \left(\alpha_{2} a\right)}{2 \cos (\beta a)}\right\} \cos (\beta z)\right] .
\end{gathered}
$$

In the case of a radially symmetric disturbance, continuity of the medium demands that the radial displacements at the line of symmetry must be zero. From the equation (2) it is obvious since $u=0 \quad$ when $r=0$.

\subsection{Impervious surface}

From the equation (4), we have $\frac{\partial s}{\partial z}=\left[A_{1} D_{3} \alpha_{1} \sin \left(\alpha_{1} z\right)+B_{1} D_{4} \alpha_{2} \sin \left(\alpha_{2} z\right)\right] J_{0}(k r) e^{i p t}$. Invoking the third boundary condition, we obtain

$$
\frac{A_{1}}{B_{1}}=\frac{-D_{4} \alpha_{2} \sin \left(\alpha_{2} a\right)}{D_{3} \alpha_{1} \sin \left(\alpha_{1} a\right)}
$$

Substitution of (11) in the first boundary condition yields

$$
\frac{C_{1}}{B_{1}}=\frac{-D_{4}\left(D_{1}+D_{3}\right) \alpha_{2} \cos \left(\alpha_{1} a\right) \sin \left(\alpha_{2} a\right)}{2 D_{3} N \beta k^{2} \sin \left(\alpha_{1} a\right) \cos (\beta a)}+\frac{\left(D_{2}+D_{4}\right) \cos \left(\alpha_{2} a\right)}{2 N \beta k^{2} \cos (\beta a)} .
$$

Substituting (11) and (12) in the expression for $\sigma_{r r}+s$, we obtain

$$
\begin{aligned}
\sigma_{r r}+s= & B_{1}\left[\frac{D_{4}\left(D_{3}+D_{7}\right) \alpha_{2} \sin \left(\alpha_{2} a\right) \cos \left(\alpha_{1} z\right)}{D_{3} \alpha_{1} \sin \left(\alpha_{1} a\right)}-\left(D_{4}+D_{8}\right) \cos \left(\alpha_{2} z\right)+\right. \\
& \left.\left\{\frac{D_{4}\left(D_{1}+D_{3}\right) \alpha_{2} \cos \left(\alpha_{1} a\right) \sin \left(\alpha_{2} a\right)}{2 D_{3} \alpha_{1} \cos (\beta a) \sin \left(\alpha_{1} a\right)}-\frac{\left(D_{2}+D_{4}\right) \cos \left(\alpha_{2} a\right)}{2 \cos (\beta a)}\right\} \cos (\beta z)\right] \\
& J_{0}(k r) e^{i p t}+B_{1} N k^{2}\left[\frac{D_{4} \alpha_{2} \sin \left(\alpha_{2} a\right) \cos \left(\alpha_{1} z\right)}{D_{3} \alpha_{1} \sin \left(\alpha_{1} a\right)}-\cos \left(\alpha_{2} z\right)+\right. \\
& \left.\left.\left\{\frac{-D_{4}\left(D_{1}+D_{3}\right) \alpha_{2} \cos \left(\alpha_{1} a\right) \sin \left(\alpha_{2} a\right)}{2 D_{3} N k^{2} \alpha_{1} \sin \left(\alpha_{1} a\right) \cos (\beta a)}+\frac{\left(D_{2}+D_{4}\right) \cos \left(\alpha_{2} a\right)}{2 N k^{2} \cos (\beta a)}\right\} \cos (\beta z)\right\}\right] \\
& J_{2}(k r) e^{i p t} .
\end{aligned}
$$

Letting $r \rightarrow 0$ in the expressions for $\sigma_{r r}+s$ and $\sigma_{r z}$, we obtain

$$
\sigma_{r z}=0, \quad \sigma_{r r}+s=B_{1} F_{2} e^{i p t},
$$

where

$$
\begin{gathered}
F_{2}=\left[\frac{D_{4}\left(D_{3}+D_{7}\right) \alpha_{2} \sin \left(\alpha_{2} a\right) \cos \left(\alpha_{1} z\right)}{D_{3} \alpha_{1} \sin \left(\alpha_{1} a\right)}-\left(D_{4}+D_{8}\right) \cos \left(\alpha_{2} z\right)+\left\{\frac{D_{4}\left(D_{1}+D_{3}\right) \alpha_{2} \cos \left(\alpha_{1} a\right) \sin \left(\alpha_{2} a\right)}{2 D_{3} \alpha_{1} \sin \left(\alpha_{1} a\right) \cos (\beta a)}\right.\right. \\
\left.\left.-\frac{\left(D_{2}+D_{4}\right) \cos \left(\alpha_{2} a\right)}{2 \cos (\beta a)}\right\} \cos (\beta z)\right] .
\end{gathered}
$$




\section{Numerical Results and Discussion}

Now we introduce non-dimensional parameters to compute the quantities $F_{1}$ and $F_{2}$ for a non-dissipation case (that is when $b=0$ ), which are approximations for the $\sigma_{r r}+s$ in the case of pervious surface and impervious surface, respectively as follows:

$$
\begin{aligned}
& a_{1}=\frac{P}{H}, a_{2}=\frac{Q}{H}, a_{3}=\frac{R}{H}, a_{4}=\frac{N}{H}, \\
& d_{1}=\frac{\rho_{11}}{\rho}, d_{2}=\frac{\rho_{12}}{\rho}, d_{3}=\frac{\rho_{22}}{\rho}, \\
& \tilde{x}=\left(\frac{V_{0}}{V_{1}}\right)^{2}, \tilde{y}=\left(\frac{V_{0}}{V_{2}}\right)^{2}, \tilde{z}=\left(\frac{V_{0}}{V_{3}}\right)^{2}, m=\frac{c}{c_{0}}, \tau \equiv \frac{p}{c_{0}}=m k,
\end{aligned}
$$

where $m$ is non-dimensional phase velocity, $\tau$ is non-dimensional frequency, and

$$
\rho=\rho_{11}+2 \rho_{12}+\rho_{22}, H=P+2 Q+R, c=\frac{p}{k}, c_{0}^{2}=\frac{N}{\rho}, V_{0}^{2}=\frac{H}{\rho} .
$$

Using non-dimensional variables defined in (16) into $F_{1}$ and $F_{2}$ given by (10) and (15), respectively, one obtains an explicit relation between non-dimensional quantities $F_{i} / F_{0} \quad\left(i=1,2, F_{0}=H k^{2}\right)$ and $z / a$ for given materials, keeping nondimensional frequency $\tau a$ and non-dimensional wavenumber $k a$ fixed. Three sets of material parameters are employed for computational work, which are presented in the table I. Of three, first two are given by Biot (Biot 1956) and third set is pertaining to sandstone saturated with kerosene given by Fatt (Fatt 1957). In material-II, mass coupling parameter is present while in material-III, elastic parameters (Lame constants) are dominant. For materials I \& II Poisson ratio is 0.47 whereas for material-III it is 0.25 .

Table 1. Three sets of material parameters employed for computational work

\begin{tabular}{|l|c|c|c|c|c|c|c|c|c|c|}
\hline Material & $a_{1}$ & $a_{2}$ & $a_{3}$ & $a_{4}$ & $d_{1}$ & $d_{2}$ & $d_{3}$ & $\tilde{x}$ & $\tilde{y}$ & $\tilde{z}$ \\
\hline I & 0.61 & 0.0425 & 0.305 & 0.034193 & 0.5 & 0 & 0.5 & 1.671 & 0.812 & 14.623 \\
\hline II & 0.61 & 0.0425 & 0.305 & 0.034193 & 0.65 & -0.15 & 0.65 & 2.388 & 0.909 & 18.002 \\
\hline III & 0.843 & 0.065 & 0.028 & 0.234 & 0.901 & -0.001 & 0.101 & 0.999 & 4.763 & 3.851 \\
\hline
\end{tabular}

Quantity $F_{i} / F_{0}$ computed against $z / a$ for three given materials, each for pervious boundary and impervious boundary. Computations are performed for various values of $k a$ and $\tau a$ as taken by Davids et.al (Davids \& Kumar 1957), which are given as follows: $k a=0.5, \tau a=0.83$, say, set $1 ; k a=1, \tau a=1.64$, say ,set 2 ; $k a=1.4, \tau a=2.13$, say, set 3.Numerical results are presented graphically in figures 1-4. All the curves are symmetric with respect to y-axis. For the material -I and material -II, all the values in the case of pervious and impervious surfaces are negative, which correspond to tension, whereas for material -III, in the case of pervious surface, some values are negative and some values are positive. Positive values correspond to stress. It is interesting to note that in the case of material -III and impervious surface, all the values are positive. From the Fig.1, it is clear that tension decreases as $k a$ and $\tau a$ increase, but in the neighbourhood of $z / a=0$ the trend is reversed. When $k a$ and $\tau a$ are high, that is in the case of set 3, the values of material-I and that of material-II are coinciding, which means that mass coupling parameter does not have any influence when $k a$ and $\tau a$ are relatively higher. From the Fig.2, it is seen that in the case of material-I, the values of pervious surface and the values of impervious surface are closer which is not the true in the case of material-II which can be seen from Fig. 3. Therefore, from Fig.2 and Fig.3, one can infer that in the case of material-II, the values are affected by the nature of the surface. It is the mass coupling parameter present in material-II is making above distinction. Fig. 4 corresponds to material -III and it is found that impervious values are higher than that of pervious values. In either case the values decrease as $k a$ and $\tau a$ increase. In the case of pervious surface the curves are concave upwards and in the case of impervious surface the curves are concave downwards. 


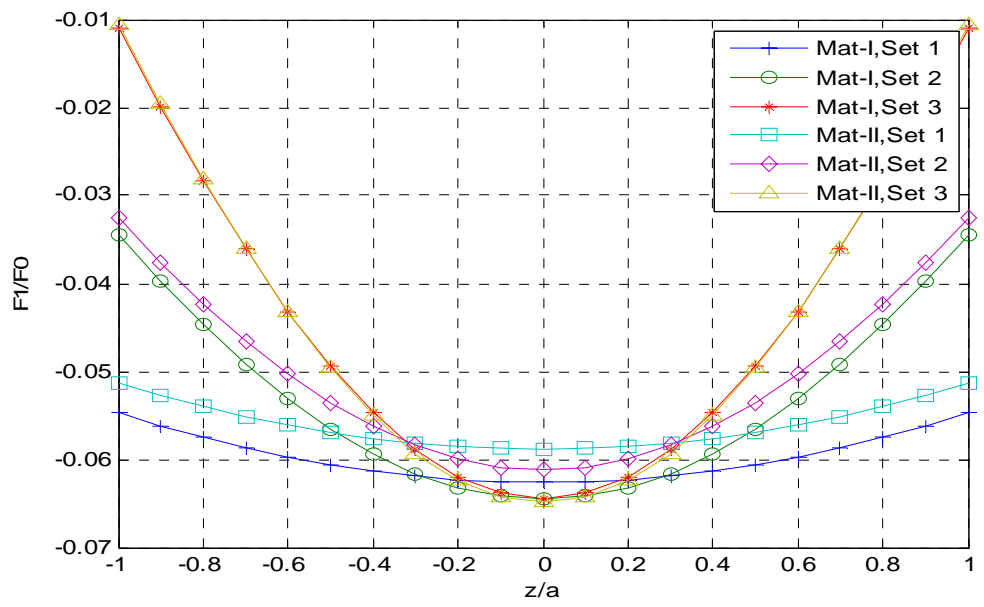

Figure1. Variation of $F_{i} / F_{0}$ with z/a in Material -I \& Material-II for Pervious Surface

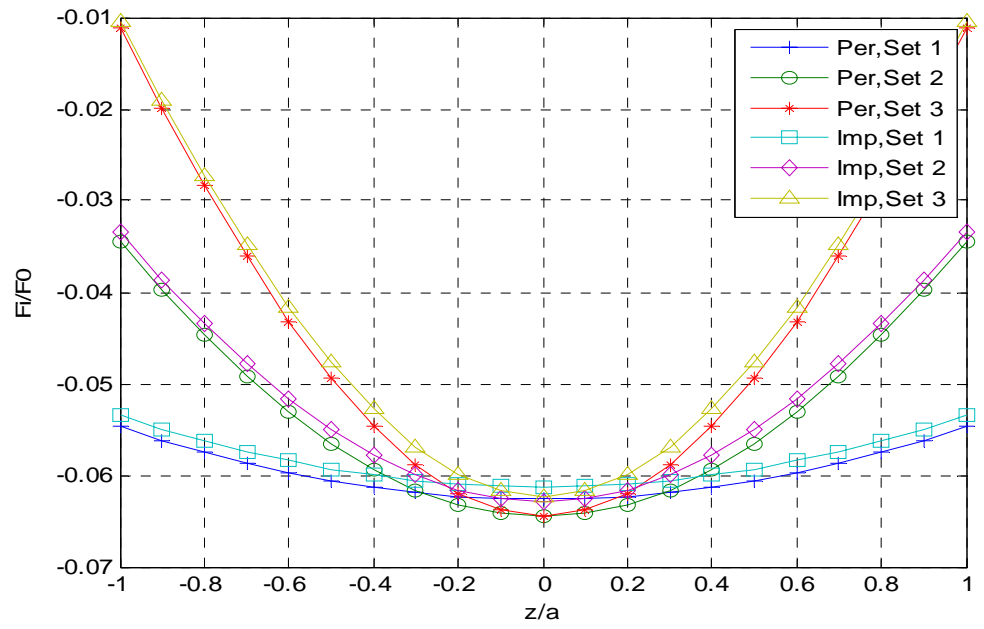

Figure 2. Variation of $F_{i} / F_{0}$ with z/a in Material -I for both Pervious and Impervious Surfaces

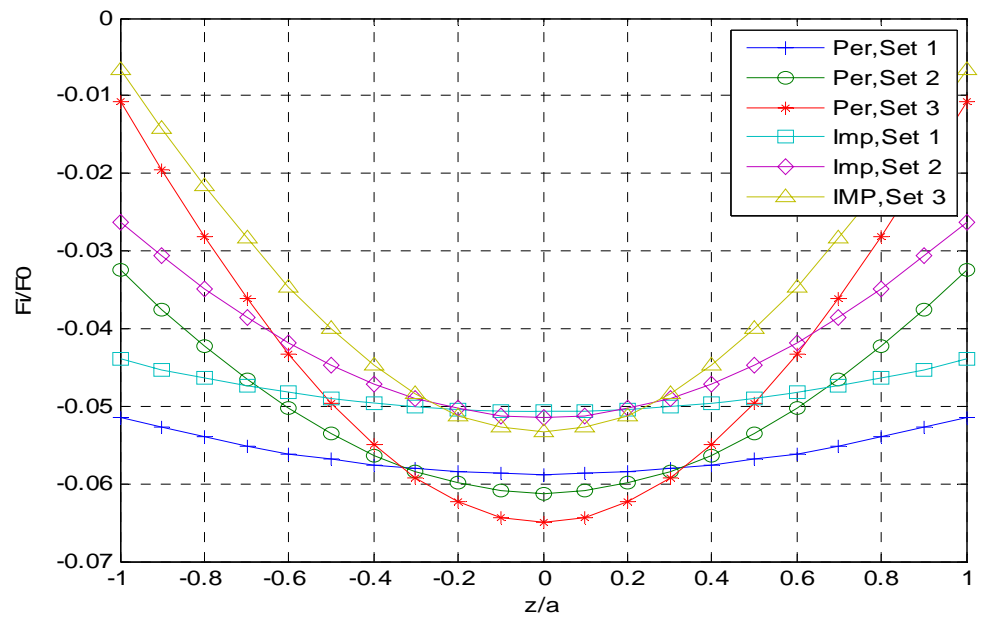

Figure3. Variation of $F_{i} / F_{0}$ with z/a in Material -II for both Pervious and Impervious Surfaces 


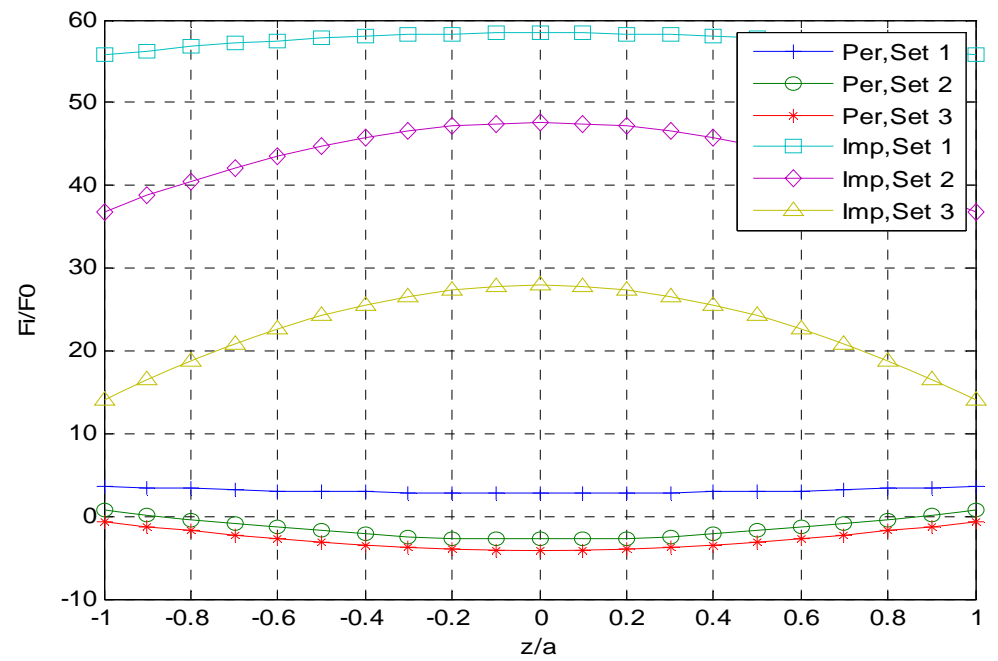

Figure4. Variation of $F_{i} / F_{0}$ with z/a in Material -III for both Pervious and Impervious Surfaces

\section{Conclusion}

The study of stress waves in a poroelastic flat slab under an excitation is made using Biot's theory. It is seen that how the radial stress components are affected in view of the traction free boundary conditions on the surfaces $Z=-a$ and $Z=a$.Our analysis is confined to a periodic disturbance, however, because of the Fourier integral theorem, it is possible to extend the conclusions to pulse propagation as well. The radial normal stress at the centre is computed against thickness of the slab for three types of materials. Numerical results show the following conclusions:

1. All the curves are symmetric with respect to y-axis.

2. The values pertaining to material-I and material-II are negative, which correspond to tension whereas the values of material-III in the case of impervious surface are positive that correspond to stress.

3. Mass coupling parameter does not affect the values when both wavenumber and frequency are high.

4. Nature of surface influences the values in presence of mass coupling parameter and impervious surface values are greater than that of pervious surface when elastic constants are higher.

\section{References}

Biot, M.A., Theory of propagation of elastic waves in fluid-saturated porous solid, J.Acoust. Soc.Am., 28, 1956, pp.168-178.

Fatt, I., The Biot-Willis elastic coefficients for a sandstone, ASME, J.Appl.Mech., 26, 1957, pp.296-297

P.Malla Reddy and M.Tajuddin, Exact analysis of the plane strain vibrations of thick-walled hollow poroelastic cylinders, Int. J. Solids and Structures, Vol.37, 2000, pp.3439-3456.

P. Malla Reddy and M.Tajuddin, Cylindrical stress waves in poroelastic flat slabs, J. Mechanics, Vol.22, 2, 2006, pp.161-165. Norman Davids and Sudheer Kumar, Cylindrical Stress Waves in Flat Slabs, 10, 4, Quart. Journ. Mech. and Applied Math.,1957, pp.465-481.

M.Tajuddin, and G. Narayan Reddy, Effect of boundaries on the dynamic interaction of a liquid filled porous layer and a supporting continuum, Sadhana, Vol. 30, part 4, 2005, pp.527-535.

M.Tajuddin and S.A. Shah, Circumferential waves of infinite poroelastic cylinders, Trans. ASME, J. Appl. Mech., Vol.73, 2006, pp.705-708.

M.Tajuddin and S.A. Shah, On torsional vibrations of infinite hollow poroelastic cylinders, J. Mech. Materials and Structures, Vol. 2, 1, 2007, pp.189-200.

\section{Acknowledgement}

This paper is presented in the $5^{\text {th }}$ National Conference on Applicable Mathematics in Wave Mechanics and Vibrations (WMVC2010) held at Kakatiya University, Warangal, India,13-15 March,2010. 


\section{Biographical notes}

Ms. B. Sandhya Rani received M. Sc. from Kakatiya University, Warangal , India in 2008. Currently, she is working as a project fellow in a major research project funded by University Grants Commission (UGC), Government of India. She has one publication in a referred international journal. Her current area of research includes wave propagation in porous materials.

Mr. T.Ramesh is a senior lecturer in the department of Mathematics, Kakatiya Degree College, Warangal, India. He has more than 20 years of experience in teaching.

Dr. P. Malla Reddy is Associate Professor and Chairman, Board of Studies, in the Department of Mathematics, Kakatiya University, Warangal, India. He has more than 15 years of experience in teaching, research and industry. His current areas of research includes wave propagation in porous materials, and internet traffic medelling-performance analysis of router. He has published about twenty papers in referred national and international journals. He has also presented several papers in national and international conferences. He is currently dealing with few projects sponsored by government of India.

Received September 2010

Accepted February 2011

Final acceptance in revised form March 2011 